\title{
A!
}

This is an electronic reprint of the original article.

This reprint may differ from the original in pagination and typographic detail.

Karki, Sabin Kumar; Ala-Laurinaho, Juha; Zheng, Jianfang; Lahti, Markku; Viikari, Ville

\section{Millimeter-wave stepped series array with LTCC}

Published in:

2019 49th European Microwave Conference, EuMC 2019

DOI:

10.23919/EuMC.2019.8910765

Published: 01/10/2019

Document Version

Peer reviewed version

Please cite the original version:

Karki, S. K., Ala-Laurinaho, J., Zheng, J., Lahti, M., \& Viikari, V. (2019). Millimeter-wave stepped series array with LTCC. In 2019 49th European Microwave Conference, EuMC 2019 (pp. 956-959). [8910765] IEEE.

https://doi.org/10.23919/EuMC.2019.8910765

This material is protected by copyright and other intellectual property rights, and duplication or sale of all or part of any of the repository collections is not permitted, except that material may be duplicated by you for your research use or educational purposes in electronic or print form. You must obtain permission for any other use. Electronic or print copies may not be offered, whether for sale or otherwise to anyone who is not an authorised user. 


\title{
Millimeter-wave stepped series array with LTCC
}

\author{
Sabin Kumar Karki ${ }^{\# 1}$, Juha Ala-Laurinaho ${ }^{\# 2}$, Jianfang Zheng ${ }^{\# 3}$, Markku Lahti ${ }^{* \# 4}$, Ville Viikari ${ }^{\# 5}$ \\ \#Department of Electronics and Nanoengineering, Aalto University, Finland \\ *VTT Technical Research Centre of Finland, VTT, Finland \\ $\left\{{ }^{1}\right.$ sabin.karki, ${ }^{2}$ juha.ala-laurinaho, ${ }^{3}$ jianfang.zheng, ${ }^{5}$ ville.viikari $\} @$ aalto.fi, ${ }^{4}$ markku.lahti@ vtt.fi
}

\begin{abstract}
The series fed antenna arrays are widely used to minimize the feeding losses. A novel method to control the coupling/admittance between the series array elements is studied. In this work, two substrate integrated waveguide (SIW) fed aperture coupled $1 \times 4$ patch arrays are designed on the LTCC platform. The admittance of the array elements are controlled with the stepped SIW and the non-uniform aperture coupling technique. The simulated and measured -10-dB impedance bandwidth of the designed antenna is approx. 6-7\%. At 75 $\mathrm{GHz}$, the maximum boresight directivity and realized gain of the designed antennas are $12 \mathrm{~dB}$ and $9 \mathrm{dBi}$, respectively. Additionally, a microstrip line (MSL)-SIW vertical transition structure is designed to facilitate the multi-layered RF structure in LTCC.
\end{abstract}

Keywords - LTCC, SIW, series array, transverse slot, vertical transition, stepped waveguide

\section{INTRODUCTION}

Millimeter-wave technology is considered as an enabler for the next generation of cellular communication system, 5G [1]. Highly directional antennas with beam steering capability are essential to counter the high propagation loss at millimeter-wave band and cover wide steering range. The series array together with the beamforming networks such as Rotman lens or butler matrix is an appropriate solution for the millimeter-wave communication system [2].

The feed line losses of an array can be reduced using series feed that minimizes the feed line length [3]. The microstrip transmission lines (MSL) are popular due to ease of manufacturing and integrating active components. However, at high frequency, the losses in MSL are high. Recently, the substrate integrated waveguide (SIW) has gained popularity at millimeter-wave frequencies due to low attenuation [4]. The fabrication of SIW is enabled by multilayer technologies such as Low Temperature Co-fired Ceramics Technology (LTCC) [5]. The LTCC technology enables the design of multilayered structures and also the integration of active components with antennas [6]. In order to design the compact multi-layered RF structure, the vertical transitions between the stacked layers are essential.

The waveguide based longitudinal and transverse slot series array architectures are widely studied, e.g. in [7]. The longitudinal or transverse slots are placed along the broad wall of a waveguide at $\lambda_{0} / 2$ separation. The slots are resonant structures i.e. the length of the slots is equal to $\lambda_{g} / 2$, therefore, the slots are inherently narrowband. In order to improve the bandwidth, the slots are used to feed/excite radiating elements like patch, resonator, magnetoelectric dipole

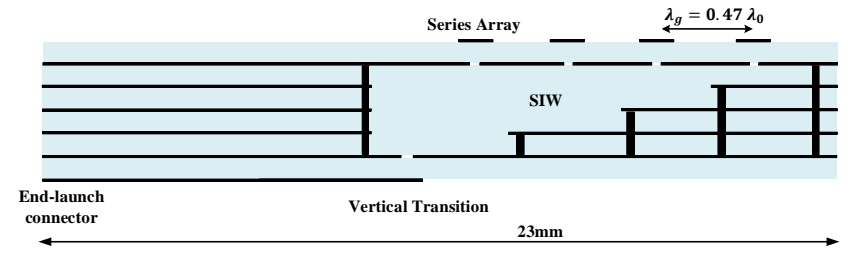

(a)

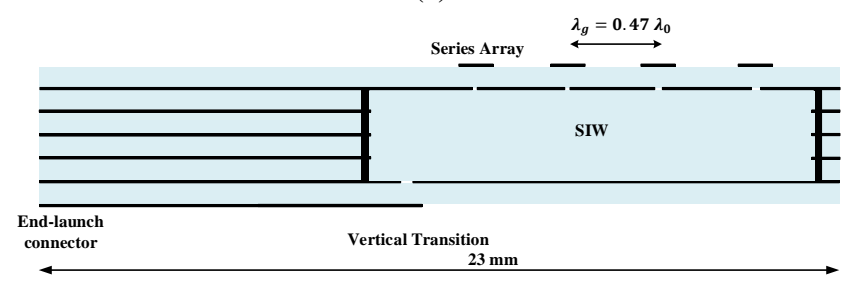

(b)

Fig. 1. Schematics of (a) the stepped-SIW fed and (b) the uniform-SIW fed array with the end-launch connector.

or Yagi-like elements [8], [9]. In general, the admittance of the longitudinal slots are controlled by the offset position from the waveguide axis. The limited ways to control the admittance of the transverse slots make the configuration less suitable compared to the longitudinal slots [10]. The amplitude of an array elements are mostly desired to be equal, however, in some cases the amplitude of the elements are optimized to generate tailored radiation pattern.

In this work, the possibility of using the height of the SIW to control the admittance of the series transverse slot fed array is studied. $1 \times 4$ stepped-SIW feed aperture coupled microstrip patch antenna arrays are designed on the LTCC platform. The performance of the stepped series array is compared with another series array designed with the non-uniform aperture coupling technique. The structures are shown in Fig. 1. The radiation pattern and low sidelobe level are considered as performance indicators that verify equal coupling between array elements. Additionally, a wideband aperture coupled vertical transition between MSL and SIW is designed to make the structure compact.

\section{SERIES ARRAY}

The admittance of the radiating slot in a waveguide is proportional to the current density, size of the slot, and the offset position of the slot from the axis of the waveguide [11]. In this work, the admittance of the coupling slot is controlled by maneuvering the current density and the size of the aperture. Two $1 \times 4$ series arrays are designed by using stepped SIW and 
unequal size of coupling slots. The series arrays are designed to operate at lower E-band dedicated for communications i.e. 71-76 GHz. The series arrays are designed on Ferro-A6M-E LTCC substrate $\left(\epsilon_{r}=5.7 \pm 0.2\right.$ and $\left.\tan \delta=0.002\right)$. The layer thickness of each LTCC dielectric layer is $0.092 \mathrm{~mm}$. The thick-film gold material, with $\sigma=7 \times 10^{6} \mathrm{~S} / \mathrm{m}$ and $5 \mu \mathrm{m}$ thickness, is used as the conductor.

The array elements are desired to be $0.5 \lambda_{0}$ apart. Since, the guided wavelength $\lambda_{g}$ inside the waveguide is different to $\lambda_{0}$, the in-phase excitation of the radiating elements becomes challenging. The guided wavelength $\lambda_{g}$ inside the SIW is determined by the substrate dielectric constant, $\epsilon_{r}$ and the width of the SIW. The width of the SIW is chosen to be 2 $\mathrm{mm}$ so that the resulting $\lambda_{g}=1.9 \mathrm{~mm}$ which is equivalent to $0.47 \lambda_{0}$. The vias of $100 \mu \mathrm{m}$ in diameter are placed at the periodic interval of $250 \mu \mathrm{m}$ to prevent leakage from the SIW.

\section{A. SIW fed Microstrip Patch Antenna}

A SIW-coupled microstrip patch antenna is designed as shown in Fig 2. The coupling slot at the center of the radiating element is placed at $\lambda_{g} / 2$ distance from the short circuit SIW wall where the transverse surface current is maximum in the waveguide. The length of the slot $l a$ and the length of the patch $l p$ are optimized to achieve resonance at desired frequency range. The $-10-\mathrm{dB}$ bandwidth of the single transverse slot fed patch is approx. $2 \mathrm{GHz}$ between $73.5-75.5 \mathrm{GHz}$, see Fig. 3(a). The maximum directivity of the single element is $7 \mathrm{~dB}$. The E-plane and H-plane directivity radiation patterns of the patch are shown in Fig. 3(b).

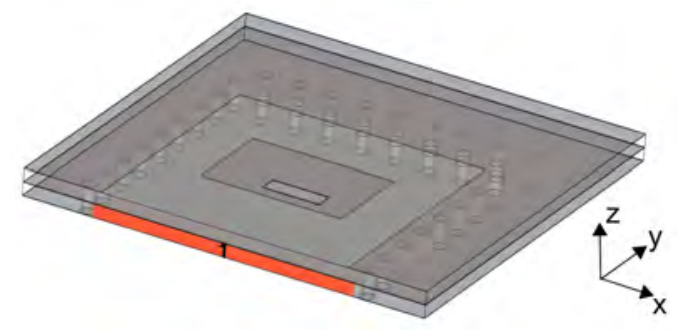

Fig. 2. A SIW-coupled microstrip patch antenna.

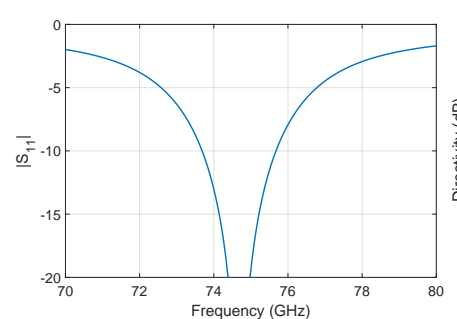

(a)

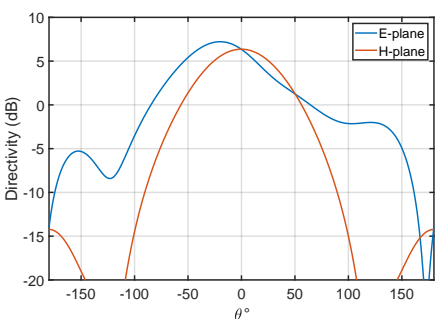

(b)
Fig. 3. (a) The reflection coefficient and (b) the radiation pattern (directivity) of the single SIW coupled microstrip patch antenna.

\section{B. Stepped series array}

At resonance, the conductance of the transverse slot is inversely proportional to the height of the waveguide [12]. As the height of the SIW increases, the current density in the broad walls of the SIW decreases and hence, the admittance to the patch decreases. Therefore, the coupling slots and patches of the same dimensions are placed at $\lambda_{g}$ distance with variable SIW height to control the admittance. The multilayered architecture of the LTCC is utilized to design the stepped SIW. In this work, the equal power is coupled to each radiating patch of the series array by linearly varying the height from one to four LTCC layers i.e. 0.092 to 0.368 $\mathrm{mm}$. The side-view of the stepped series arrays fed with the end-launch connector and the GSG-probe feed is shown in Fig. 1 (a) and 4 (a), respectively. The detailed dimensions of the designs are summarized in Table 1. Additionally, the height of the SIW can be varied according to the required excitation of the array element.

Table 1. Dimensions (in $\mathrm{mm}$ ) of the designed series arrays.

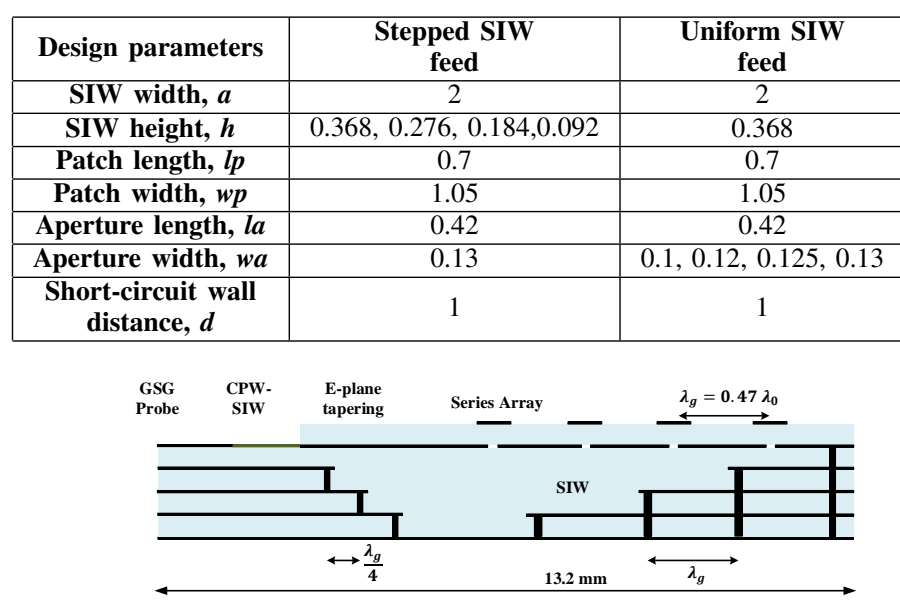

(a)

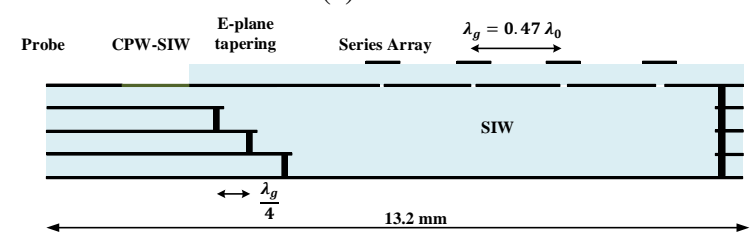

(b)

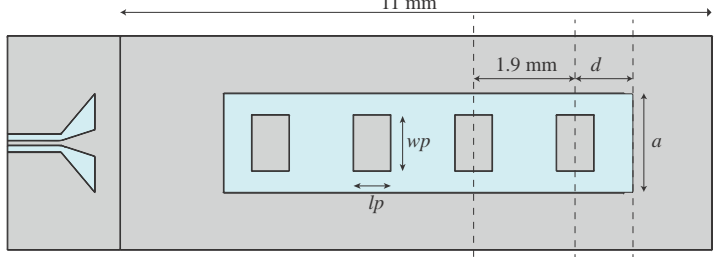

(c)

Fig. 4. Schematics of (a) the stepped-SIW fed and (b) the uniform-SIW fed array, and (c) the top view of the array with the GSG-probe connection.

\section{Uniform series array}

The schematics of the uniform SIW fed series array based on non-uniform slot dimensions are shown in Fig. 1 (b) and 4(b). In this design, the variable slot widths are used to control the coupling to the array elements. The length of the patch $l p$ and the length slot $l a$ determines the resonant frequency of the structure. The width of the slot is carefully optimized for equal power coupling to array elements while the height of SIW is kept uniform i.e. $h=0.368 \mathrm{~mm}$, through the length of 
the array. The smallest difference between the slot width of the series array elements ranges between 5-20 $\mu \mathrm{m}$. Manufacturing the antennas with such precision is demanding for available technologies.

The designed series antennas are fed in two different ways: (i) MSL-SIW vertical transition, together with end-launch connector and (ii) E-plane taper SIW together with GSG-probe, as illustrated in [13].

\section{MSL-SIW VERTICAL TRANSITION}

The designed transition integrates a MSL and SIW in adjoining stacked layers of the LTCC, see Fig. 5 (a) and 6 (a). An aperture in the common ground plane of the MSL and SIW is used to couple the field. The operation frequency of the transition is determined by the width of the SIW and the length of the slot. The slot is placed at approx. $\lambda_{g} / 4$ distance before the end of the MSL, so that the current is maximum at the slots and the maximum power is coupled into SIW. Similarly, distance between the slots and the SIW via wall is $\lambda_{g} / 4$, so that the reflected field adds constructively in the forward direction.

The vertical transition structure is designed to operate in lower E-band i.e. (71-76) $\mathrm{GHz}$, dedicated to telecommunications. The $\mathrm{S}$-parameter of a single transition with lossless material $(\tan \delta=0$, conductivity $\sigma=\infty)$ and realistic material $\left(\tan \delta=0.002, \sigma=7 \times 10^{6} \mathrm{~S} / \mathrm{m}\right)$ is shown in the Fig. 6 (b). At $73 \mathrm{GHz}$, with realistic material the insertion loss is $0.8 \mathrm{~dB}$ whereas with the lossless material insertion loss is $0.2 \mathrm{~dB}$. The loss $0.2 \mathrm{~dB}$ is accounted for the reflection loss and the radiation losses.

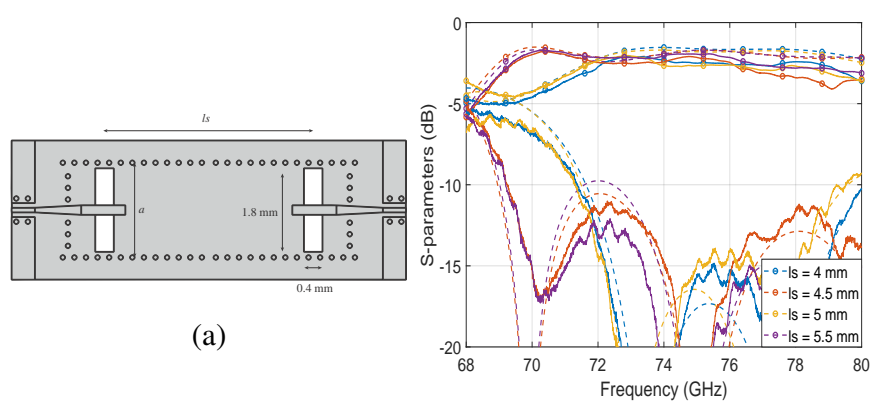

(b)

Fig. 5. (a) Bottom view, and (b) the simulated (- -) and measured (-) S-parameter i.e. $\left|S_{11}\right|(-)$ and $\left|S_{12}\right|$ (-o-), comparison of the back-to-back vertical transition structures of different lengths.

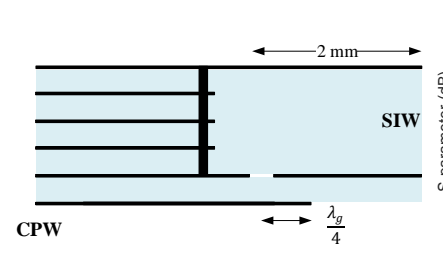

(a)

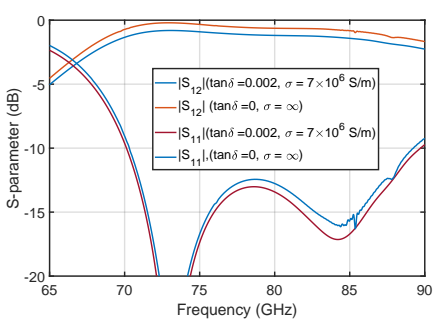

(b)
Fig. 6. (a) Orthographic view, and (b) simulated S-parameter comparison of the isolated vertical transition structure.

For the experimental verification, four back-to-back vertical transition structures with $4,4.5,5$ and $5.5 \mathrm{~mm}$ of
SIW lengths are manufactured, see Fig. 5(a). The comparison between the simulated and measured $\mathrm{S}$-parameter of the back-to-back vertical transition structure is shown in the Fig. 5(b). The measured reflection and transmission coefficient performance follows the simulated results. Based on the measurement results, the insertion loss of a single vertical transition is less than $1.3 \mathrm{~dB}$ within the designed frequency 71-76 GHz.

\section{SERIES ARray Measurement}

The fabricated prototypes of different versions of $1 \times 4$ series arrays are shown in Fig 7. The reflection coefficient and gain measurement of the GSG probe fed prototypes are done in millimeter wave probe station [14], whereas the end-launch connector feed prototypes are measured in the planar near field measurement facility.

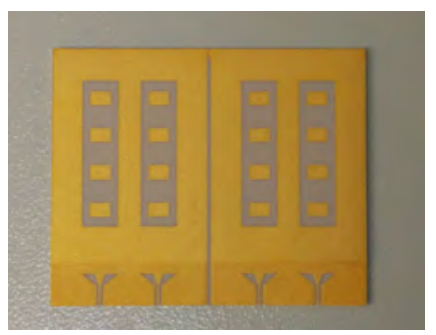

(a)

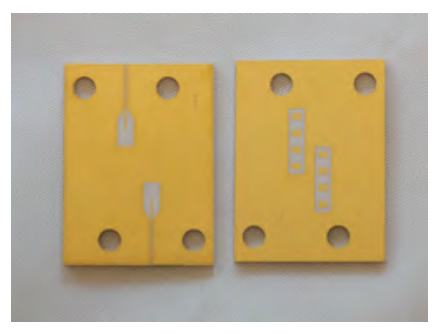

(b)
Fig. 7. (a) The GSG-probe fed, and (b) the top and bottom view of the end-launch connector fed $1 \times 4$ series array on LTCC substrate.

The simulated reflection coefficient $\left(\left|S_{11}\right|\right)$ of the designed series antenna arrays have the $-10-\mathrm{dB}$ impedance bandwidth of approx. $5 \mathrm{GHz}$ covering the designed frequency range i.e. 71-76 GHz as shown in Fig. 8(a). The measured reflection coefficients $\left|S_{11}\right|$ are marginally shifted towards higher frequency and the measured ones are larger than the simulated ones. In the case of end-launch connector fed prototypes, the simulation analysis shows that the air gap between the LTCC board and end-launch connector explains for such a difference. In the case of the GSG-probe fed prototypes, the gap between the signal line and the grounds of the CPW seems to be larger than the designed, thus being possible reason for the deviation.

The simulated peak directivity over the lower E-band is more than $10.5 \mathrm{~dB}$ and $11.7 \mathrm{~dB}$ for the end-launch feed and GSG-probe feed, respectively. Due to the longer transmission lines, the peak realized gain of the end-launch fed prototype (approx. $8 \mathrm{dBi}$ ) is approx. $1 \mathrm{~dB}$ lower than the GSG-probe prototypes (approx. $9 \mathrm{dBi}$ ). The simulation results show that the performance of the uniform and stepped series array are comparable. Analogous to the measured $\left|S_{11}\right|$, the measured realized gains are also shifted towards higher frequency. The measured realized gain of the GSG-probe feed prototypes are $1 \mathrm{~dB}$ lower than the simulated results (approx. $8 \mathrm{dBi}$ vs $9 \mathrm{dBi}$ ). The higher $\left|S_{11}\right|$ is the possible cause for the lower realized gain. In case of the end-launch connector feed prototypes, the peak measured gain is $1 \mathrm{~dB}$ higher than the simulated results. 


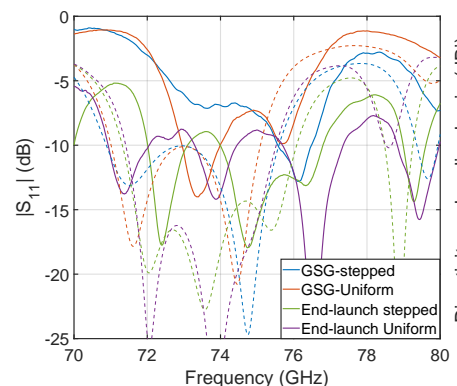

(a)

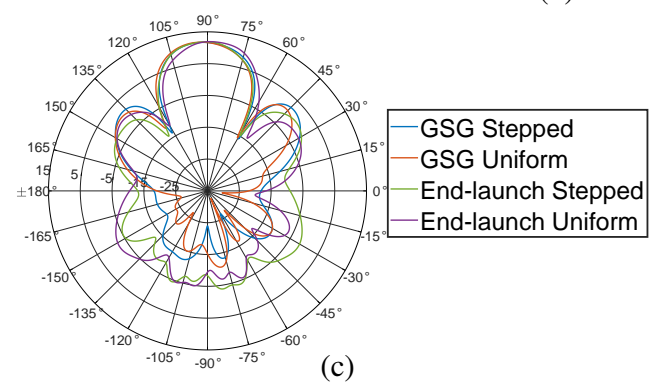

(c)

Fig. 8. (a) The simulated (- -) and measured (-) $\left|\mathrm{S}_{11}\right|$; (b) the simulated peak directivity (-.-), and realized gain (- -) and the measured realized gain (-); and (c) the simulated directivity radiation pattern comparison at $75 \mathrm{GHz}$ in E/H-plane.

The simulated mainlobe of all prototypes are oriented towards boresight, see Fig. 8(c). At $75 \mathrm{GHz}$, the simulated mainlobe directivity for all prototypes is approx. $12 \mathrm{~dB}$ and the radiation pattern is symmetric about the axis i.e. the first side lobe level (SLL) are equal. The maximum SLL of the end-launch connector and GSG-probe fed is $-12.3 \mathrm{~dB}$ and -11.7 $\mathrm{dB}$, respectively. The end-launch connector fed prototypes have higher back lobe due to the radiation from microstrip line of the vertical transition structure. Similarly, the first SLL of the CPW fed structure is slightly higher due to spurious radiation from the CPW and CPW-SIW transition.

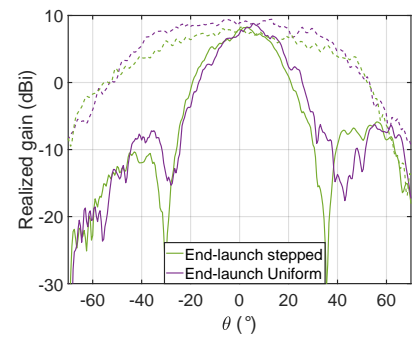

(a)

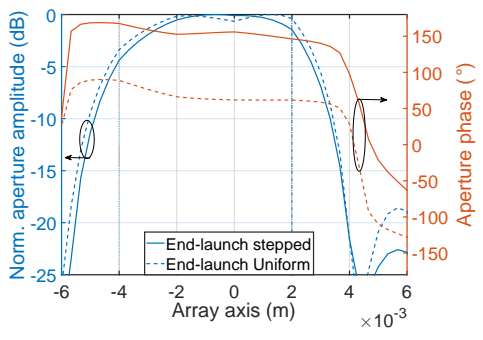

(b)
Fig. 9. (a) The measured E-plane (-) and H-plane (- -) realized gain radiation pattern, and (b) the aperture amplitude and phase along array axis of the end-launch connector fed arrays at $75 \mathrm{GHz}$.

The measured E-plane and H-plane radiation pattern of the end-launch connector fed stepped and uniform series arrays are shown in the Fig. 9(a). Within the designed frequency range, the mainlobe of both stepped and uniform array prototypes are directed towards the boresight and the peak realized gain of the uniform and the stepped series array is $8.9 \mathrm{dBi}$ and 8.5 $\mathrm{dBi}$ at $75 \mathrm{GHz}$, respectively. The measured radiation pattern is almost symmetrical about the axis and the SLLs are below $15 \mathrm{~dB}$. The measured near-field data is back propagated to the array aperture to find the radiated amplitude and phase, see
Fig. 9(b). The calculated result highlights that each radiating elements have approx. equal power and the same phase with both the feeding techniques.

\section{CONCLUSION}

In this work, two SIW feed aperture coupled $1 \times 4$ patch arrays are designed on the LTCC platform. The simulation and measurement result confirm that the admittance of SIW fed element can be controlled by maneuvring the height of the SIW. The simulation and measurement results highlight that the performance of the stepped series array is comparable with the uniform series array.

\section{ACKNOWLEDGMENT}

We would like to thank Business Finland for supporting the work through 5WAVE project.

\section{REFERENCES}

[1] T. S. Rappaport, S. Sun, R. Mayzus, H. Zhao, Y. Azar, K. Wang, G. N. Wong, J. K. Schulz, M. Samimi, and F. Gutierrez, "Millimeter wave mobile communications for 5G cellular: It will work!" IEEE Access, vol. 1, pp. 335-349, 2013.

[2] A. Lamminen, J. Säily, M. Kaunisto, M. Pokorný, J. Aurinsalo, and Z. Raida, "Gain enhanced millimetre-wave beam-switching Rotman lens antenna designs on LCP," in 2017 11th European Conference on Antennas and Propagation (EUCAP), March 2017, pp. 2781-2785.

[3] T. Metzler, "Microstrip series arrays," IEEE Trans. Antennas Propag., vol. 29 , no. 1 , pp. 174-178, January 1981.

[4] H. Uchimura, T. Takenoshita, and M. Fujii, "Development of a "laminated waveguide"," IEEE Trans. Microw. Theory Techn., vol. 46, no. 12, pp. 2438-2443, Dec 1998.

[5] F. F. Manzillo, M. Smierzchalski, M. Ettorre, J. Aurinsalo, K. T. Kautio, M. S. Lahti, A. E. I. Lamminen, J. Säily, and R. Sauleau, "An LTCC beam-switching antenna with high beam overlap for $60-\mathrm{GHz}$ mobile access points," in 2017 IEEE International Symposium on Antennas and Propagation USNC/URSI National Radio Science Meeting, July 2017, pp. 1441-1442.

[6] A. E. I. Lamminen, J. Säily, and A. R. Vimpari, "60-GHz patch antennas and arrays on LTCC With embedded-cavity substrates," IEEE Trans. Antennas Propag., vol. 56, no. 9, pp. 2865-2874, Sep. 2008.

[7] A. Stevenson, "Theory of slots in rectangular wave-guides," J. Appl. Phys., vol. 19, no. 1, pp. 24-38, 1948.

[8] N. Ghassemi and K. Wu, "High-efficient patch antenna array for E-band gigabyte point-to-point wireless services," IEEE Antennas Wireless Propag. Lett., vol. 11, pp. 1261-1264, 2012.

[9] Z. Hao, Q. Yuan, B. Li, and G. Q. Luo, "Wideband $W$-band substrate-integrated waveguide magnetoelectric (ME) dipole array Antenna," IEEE Trans. Antennas Propag., vol. 66, no. 6, pp. 3195-3200, June 2018.

[10] X. Shan, Z. Shen, and P. T. Teo, "A novel waveguide transverse slot antenna array," in IEEE Antennas and Propagation Society International Symposium (IEEE Cat. No.02CH37313), vol. 3, June 2002, pp. 420-.

[11] R. J. Stegen, "Longitudinal shunt slot characteristics," Hughes Technical Memorandum No. 261, vol. 261, no. 1, pp. 1-21, 1951.

[12] A. Oliner, "The impedance properties of narrow radiating slots in the broad face of rectangular waveguide: Part I-Theory," IRE Trans. Antennas Propag., vol. 5, no. 1, pp. 4-11, January 1957.

[13] J. Haarla, J. Ala-Laurinaho, M. Lahti, and V. Viikari, "Phased antenna array with in-line vector modulators on LTCC," IEEE Transaction on Antennas and Propagation, 2019 (in preparation).

[14] J. Zheng, J. Ala-Laurinaho, and A. V. Räisänen, "On the one-antenna gain measurement method in probe station environment at mm-wave frequencies," IEEE Trans. Instrum. Meas., 2019 (DOI: 10.1109/TIM.2018.2890454). 